\title{
Tecnologías para el aprendizaje y el conocimiento: un estudio bibliométrico
}

Diana Marín Suelves. Universitat de València

0000-0002-5346-8665

$\mathrm{M}^{\mathrm{a}}$ Isabel Vidal Esteve. Universitat de València

0000-0002-3504-8114

Recepción: 29/01/2019 | Aceptado: 12/02/2019

Correspondencia a través de ORCID: Diana Marín Suelves

iD $0000-0002-5346-8665$

Citar: Marín, D y Vidal, MI (2019). Tecnologías para el aprendizaje y el conocimiento: un estudio bibliométrico. REIDOCREA, 8(2), 37-50.

Resumen: Dado el impacto de las tecnologías en el contexto educativo, en este trabajo se pretende analizar las tendencias de la investigación realizada en el campo de las Tecnologías del Aprendizaje y el Conocimiento (TAC) a través de un análisis bibliométrico de la producción científica en la base de datos Scopus. Se incluyen un total de 59 artículos publicados entre 2002 y 2018. Los resultados indican un incremento en los últimos años de la presencia de publicaciones científicas en revistas del campo de las Ciencias Sociales, y una amplia dispersión en revistas de áreas muy dispares como Ingeniería, Matemáticas o Medicina. Los datos muestran que tanto el número de artículos como el impacto de estos siguen siendo escasos en el área de las ciencias sociales, el peso de nuestro país es preferente en este campo y finaliza este texto con algunas líneas de investigación futura.

Palabras clave: Tecnología | bibliometría

\section{Technologies for learning and knowledge: a bibliometric study}

\begin{abstract}
Due to the impact of technologies in the educational context, in this dissertation, we intend to analyze trends of research carried out in the field of Learning and Knowledge Technologies (LKT) through a bibliometric analysis of scientific production on Scopus database. An amount of 59 articles, published between 2002 and 2018, are included. The results indicate an increase in the last years of the presence of scientific publications in journals in the field of Social Sciences, and a wide dispersion in journals of very different areas such as Engineering, Mathematics or Medicine. The data show that, the number of articles and the impact of these are still scarce in the area of social sciences; the weight of our country is preferred in this field and ends this text with some proposals to lines of future investigation.
\end{abstract}

Keywords: Technology | bibliometrics

Financiación y agradecimientos: A la Conselleria de Educación, Investigación, Cultura y Deporte por la promoción de la investigación científica, el desarrollo tecnológico y la innovación en la Comunitat Valenciana. Este trabajo se basa en los resultados de la fase I del proyecto emergente titulado: Análisis de las estrategias docentes ante la digitalización de los contenidos del curriculum de educación infantil y primaria (GV/2018/074).

\section{Introducción}

Todavía hoy en día no existe un acuerdo sobre la forma de denominar la sociedad actual (Salvat y Serrano, 2011; UNESCO, 2005). Algunos como Castells (1997) hablaron de sociedad red, otros como Machlup (1962) de sociedad de la información, por su parte, Bell (1973) y Tournaire (1969) la llamaron sociedad post-industrial, mientras que Drucker (1969), la denominó de sociedad del conocimiento. La clave de todas estas denominaciones se sitúa en la importancia dada al conocimiento y la tecnología.

En la década de los 70, cuando nacieron los primeros procesadores que integraban en un solo circuito los elementos básicos del ordenador, se empezó a hablar de computadores, ordenadores y PCs. Fue entonces cuando surgió el concepto de nuevas tecnologías (NNTT) y en las escuelas todavía ni se pensaba en que aquellos dispositivos electrónicos podrían llegar algún día a las aulas. Unos 15 años después, surgieron los 
primeros proyectos experimentales relacionados con las NNTT y la educación como "Atenea" y "Mercurio", que en 1992 se integrarían en el sistema educativo en nuestro país. Con el crecimiento de Internet, a partir de los 90, adquirieron mayor valor la informática y las comunicaciones y se empezó a hablar de TIC (Tecnologías de la Información y la Comunicación). La nomenclatura cambió porque las nuevas tecnologías, como todo lo nuevo, tuvieron un carácter perecedero y dejaron de serlo, e Internet aceleró la comunicación y, consecuentemente, el flujo de información generado de manera exponencial por lo que, resultó necesario adjetivar aquellas Tecnologías (que ya no eran nuevas) con términos de actualidad, aparentemente no perecederos como son Información y Comunicación (Muñoz, 2008).

El uso de tecnología en todos los ámbitos de la vida cotidiana está conllevando nuevas exigencias sociales $y$, con ello también, nuevas demandas en el contexto educativo a las que necesariamente debemos ir adaptándonos. No cabe duda de que son múltiples los beneficios de la introducción de la tecnología que se registran en distintos campos como la docencia, investigación, gestión y transferencia (García-Peñalvo, 2018).

Actualmente, la LOMCE, Ley Orgánica 8/2013, de 9 de diciembre, para la mejora de la calidad Educativa, establece en su preámbulo (artículos IV, X y XI) distintas menciones relativas dichas Tecnologías de la Información y la Comunicación en las aulas, refiriéndose a la necesidad del uso de estas como pieza fundamental para lograr el cambio metodológico hacia una educación de calidad. Plantea, con ello, un nuevo modelo de digitalización de la escuela que se adecúe a los cambios que se han ido sucediendo en la forma de enseñar y aprender, de comunicarse y de responder a las demandas de las generaciones actuales, partiendo para ello, de la necesaria formación del profesorado.

Sin embargo, y pese a que la introducción de la tecnología en la educación no es algo reciente, son numerosos los docentes que todavía no tienen suficiente formación acerca de las TIC y de su uso adecuado en las aulas educativas (Jaén, García y Piñar, 2012; Llamas y Macías, 2018; Urueña, 2016).

Y es que, en este panorama heterogéneo y cambiante es difícil saber cómo proceder de cara a brindar una formación efectiva a los docentes que se adecúe a las necesidades tecnológicas de la sociedad actual (Poitras, Doleck, Huang y Lajoie, 2017). Lo que más frecuentemente sucede es que se da una formación docente basada en listados de herramientas o actividades aisladas, para emplearlas tal y como fueron diseñadas, y esto, aparte de que en cualquier momento quedará obsoleto, resulta carente de conocimientos metodológicos necesarios para aprender a generar, a través de estos instrumentos, aprendizajes verdaderamente significativos (Rivero, Chavez, Vasquez y Blumen, 2016). Y esta mezcla, la de la tecnología con la metodología, es lo que define las TAC (Tecnologías del Aprendizaje y el Conocimiento) según Enríquez (2012), el cambio del "aprendizaje de la tecnología" al "aprendizaje con la tecnología". Lozano (2011, p. 46) las describe como la conjunción de tecnología y metodología que "trata de orientar las tecnologías de la información y la comunicación (TIC) hacia unos usos más formativos, tanto para el estudiante como para el profesor, con el objetivo de aprender más y mejor".

Lo que subyace a esta información es que las TIC representan una herramienta de innovación educativa, pero no se busca simplemente llevar las TIC al aula e integrarlas en ella, si no que se trata de ir más allá; de lograr que estas conformen un recurso para el aprendizaje y un agente de inclusión educativa (Sancho, 2008). 
Aun así, los centros educativos se encuentran inmersos en una persistente dificultad para convertir las tecnologías de la información y la comunicación (TIC) en las TAC, puesto que, para ello, se requiere un cambio significativo en la formación inicial del profesorado y en el sistema organizativo de la enseñanza, para que sea visible en las prácticas docentes (Llamas y Macías, 2018; Spiteri y Chang, 2018). Es una prioridad, como se ha comentado, lograr la alfabetización digital de los docentes de forma urgente y precisa con el objetivo de alcanzar el uso de las TAC (Cózar, de Moya, Hernández y Hernández, 2016), que implican además de las tecnologías de la información y la comunicación, la formación pedagógica necesaria para saber emplearlas en las aulas y apostar por una metodología renovada ajustada a las características del sistema educativo vigente (Baltodano, 2018; De Oliveira y Dias, 2018).

Con el fin de estudiar con cierta profundidad esta situación, se ha considerado conveniente plantear un análisis bibliométrico sobre las tendencias de la producción científica sobre las TAC en el campo de las Ciencias Sociales.

\section{Objetivos o hipótesis}

Algunas de las preguntas que nos planteamos y a las que pretendemos dar respuesta son: ¿qué comportamiento bibliométrico siguen las publicaciones relacionadas con las TAC?, ¿qué países destacan en la literatura sobre el tema?, ¿qué posición ocupa España en cuanto a la productividad científica en el campo?, ¿qué índices de repercusión reflejan las publicaciones sobre las TAC para la comunidad científica?, ¿qué impacto tienen las TAC en las diferentes etapas educativas?

Nuestra hipótesis de partida es que las TAC son un campo de investigación en auge, con mayor número de publicaciones en los últimos años, con estudios realizados en diferentes países, sin saber qué peso tiene España, y que estas tecnologías se emplean en todas las etapas educativas.

\section{Método}

Desde una perspectiva cienciométrica se realiza este estudio bibliométrico que aporta datos sobre la actividad científica y la situación del objeto de estudio (Tomás-Gorriz y Tomás-Casterá, 2018), en este caso las TAC, y el impacto en la comunidad. Según estos autores la bibliometría consiste en analizar los rasgos cuantificables de la literatura científica. Los indicadores bibliométricos son válidos en aquellas áreas en las que las publicaciones son un resultado esencial de la investigación. Por esta razón, su validez es máxima en el estudio de las grandes áreas, donde predomina la publicación científica, como es el caso. Además, siguiendo las consideraciones de Bordons y Zulueta (1999), sobre la fiabilidad de los estudios bibliométricos según el tamaño de las unidades o del área de estudio, se ha considerado conveniente realizar un mesoanálisis, con el fin de analizar la producción sobre la temática en toda un área científica completa, las Ciencias Sociales (King, 1978).

Además, partiendo de la premisa de que las bases de datos bibliográficas son la principal fuente de información que se utiliza en los estudios bibliométricos y de que la validez de un estudio dependerá en gran medida de que la base de datos seleccionada cubra de forma adecuada el área objeto de estudio, se ha considerado pertinente emplear la base Scopus pues ha sido considerada por varios autores (Granda, et al., 2013; Hernández, Sans, Jové y Reverter, 2016) como la más completa en cobertura temporal y en número de títulos por área. 
Se ha tomado como referencia para la búsqueda la base de datos Scopus por su reconocido prestigio en el ámbito científico, y se han empleado como palabras clave: technology \& learning \& knowledge \& school \& primary or nursery.

Para el análisis de datos se ha empleado también la herramienta Vosviewer (Van Eck y Waltman, 2010) con el fin de llevar a cabo la construcción y representación gráfica de mapas de coocurrencia, con conteo fraccional, ya que ha demostrado ser preferible al completo (Perianes, Waltman y Van Eck, 2016).

Además, se han establecido una serie de indicadores de colaboración científica de primera generación propuestos por Aleixandre (2010) que se agrupan en torno a la productividad científica, colaboración, circulación, consumo y repercusión e impacto, y, por último, se han tenido en cuenta variables estructurales y de contenido, en base a categorías que han emergido de la lectura de los textos.

\section{Resultados}

En primer lugar, se han tomado como referencia para realizar el análisis de los datos obtenidos los siguientes indicadores: productividad científica, colaboración, circulación, consumo e impacto.

En cuanto a la productividad científica, se analiza, en primer lugar, el número de documentos publicados por año sin limitar el periodo temporal con el fin de estudiar la frecuencia de publicación en este tema, así como la tendencia general diacrónica. El periodo temporal del que se han obtenido resultados es de dieciséis años, entre el 2002 y el 2018, y el número de trabajos obtenidos fue de 557 y se eligieron los 74 con acceso abierto para poder realizar el análisis del contenido. De estos se descartaron 15 puesto que se consideró, tras la lectura de los títulos y los resúmenes, que no se ajustaban a la temática propuesta o que no tenían vinculación con las Ciencias Sociales.

El análisis de los datos que a continuación se realiza resulta del estudio bibliométrico de los cincuenta y nueve documentos resultantes tras la búsqueda con los criterios especificados anteriormente.

En la Figura 1 se puede comprobar, siguiendo la línea de tendencia destacada en azul, como el número de obras publicadas ha ido incrementando a lo largo de los años de forma paulatina pero no uniforme. En un periodo inicial, se registran muy pocos documentos, uno en 2002 (Chambers, 2002, que pese a contener las palabras clave no contempla las TAC como se ha definido en la introducción, sino que se centra en la tecnología para el futuro escolar) y ninguno en los tres años siguientes, tampoco en 2008. Posteriormente, se constata un incremento de las publicaciones alcanzando un máximo relativo en 2010 con cinco documentos (Altimparmak y Yazici, 2010; Kasapoğlu, 2010; Leung, 2010; Prendes, Castañeda y Gutiérrez, 2010; Rohaan, Taconis y Jochems, 2010; Shahali y Halim, 2010), de nuevo se origina un periodo de decrecimiento hasta 2012; momento en el que vuelve a repuntar la investigación hasta alcanzar en 2014 la cifra de nueve artículos (Chiphiko y Shawa, 2014; Gill, Dalgarno y Carlson, 2014; Hung, Kuo, Sun y Yu, 2014; Lumadi, 2014; Miller et al., 2014; Ojo, Abayoi y Odozi, 2014; Orlando, 2014; Pareto, 2014; Vyas, 2014), el mayor número de publicaciones registradas, equivalentes a las del 2018 (Baltodano, 2018; Brulé y Bailly, 2018; Mikerova, Sergeeva, Mardirosova, Kazantseva y Karpenko, 2018; Mori y Shiogama, 2018; De Oliveira y Dias, 2018; Spiteri y Chang, 2018; Tärning, 2018; Reddy et al., 2018; Phungsuk, Viriyavejakul y Ratanaolarn, 2018). 


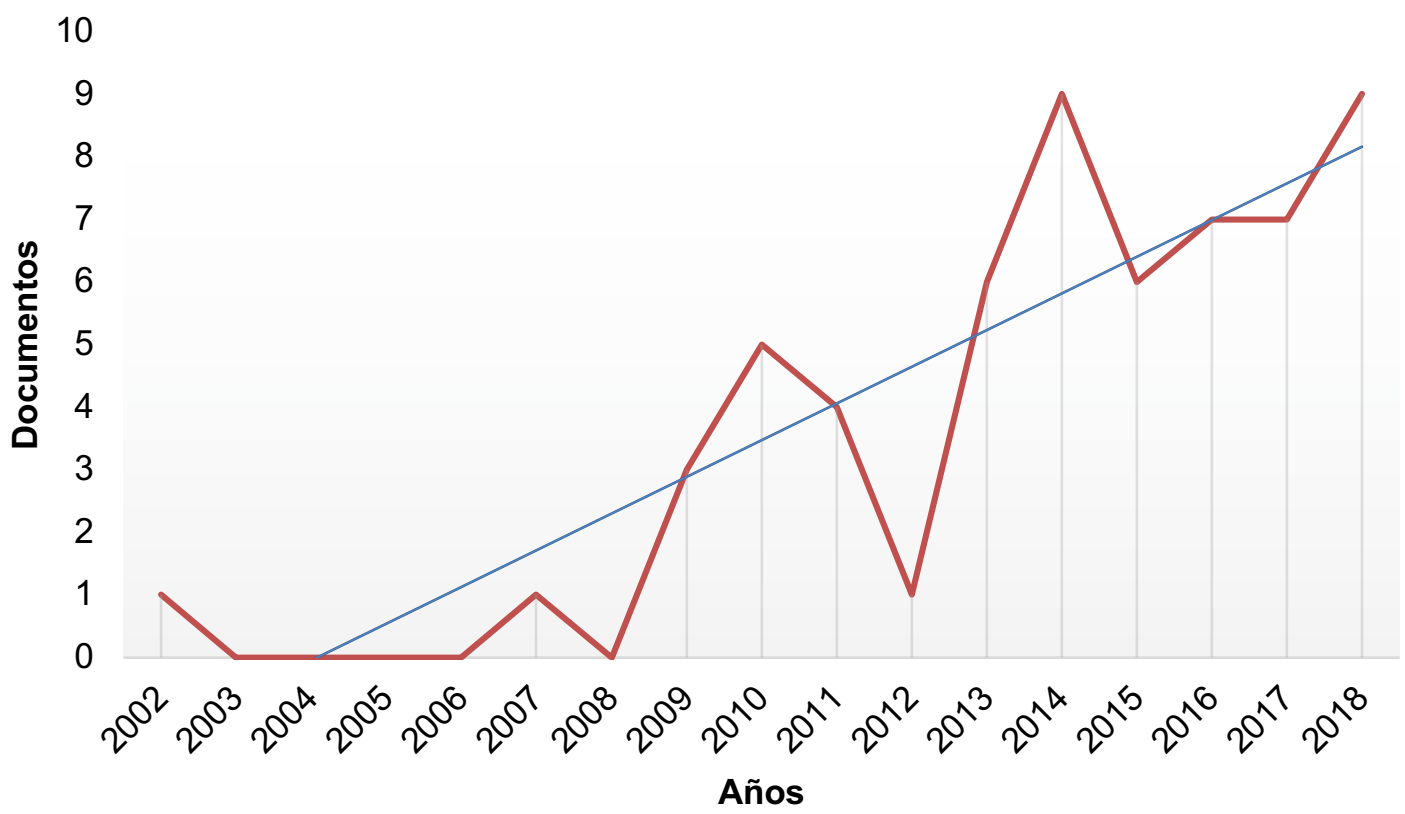

Figura 1. Frecuencia anual de publicaciones en Scopus y tendencia.

Por otra parte, en la Figura 2 se procede a analizar el tamaño bibliométrico de cada uno de los países en los que se registran publicaciones sobre el tema en el periodo estudiado. El reparto sobre el gráfico de sectores se observa bastante equilibrado, destacando tres países: China, España y Turquía, con el mayor número de publicaciones, seis cada uno, seguidos por Estados Unidos y Reino unido con cinco y cuatro documentos respectivamente, posteriormente encontramos países como: Australia, Países bajos, Sudáfrica, Suecia y Taiwán con tres publicaciones cada uno, y Canadá, India, Singapur y Tailandia, se sitúan con dos documentos. Quedan, además, un grupo de países con menor representación, únicamente un documento en cada uno de ellos, y que no quedan contemplados en el gráfico, como son: Austria, Brasil, Costa Rica o Chipre. Es de destacar que España se encuentre entre los países punteros con seis trabajos publicados recientemente.

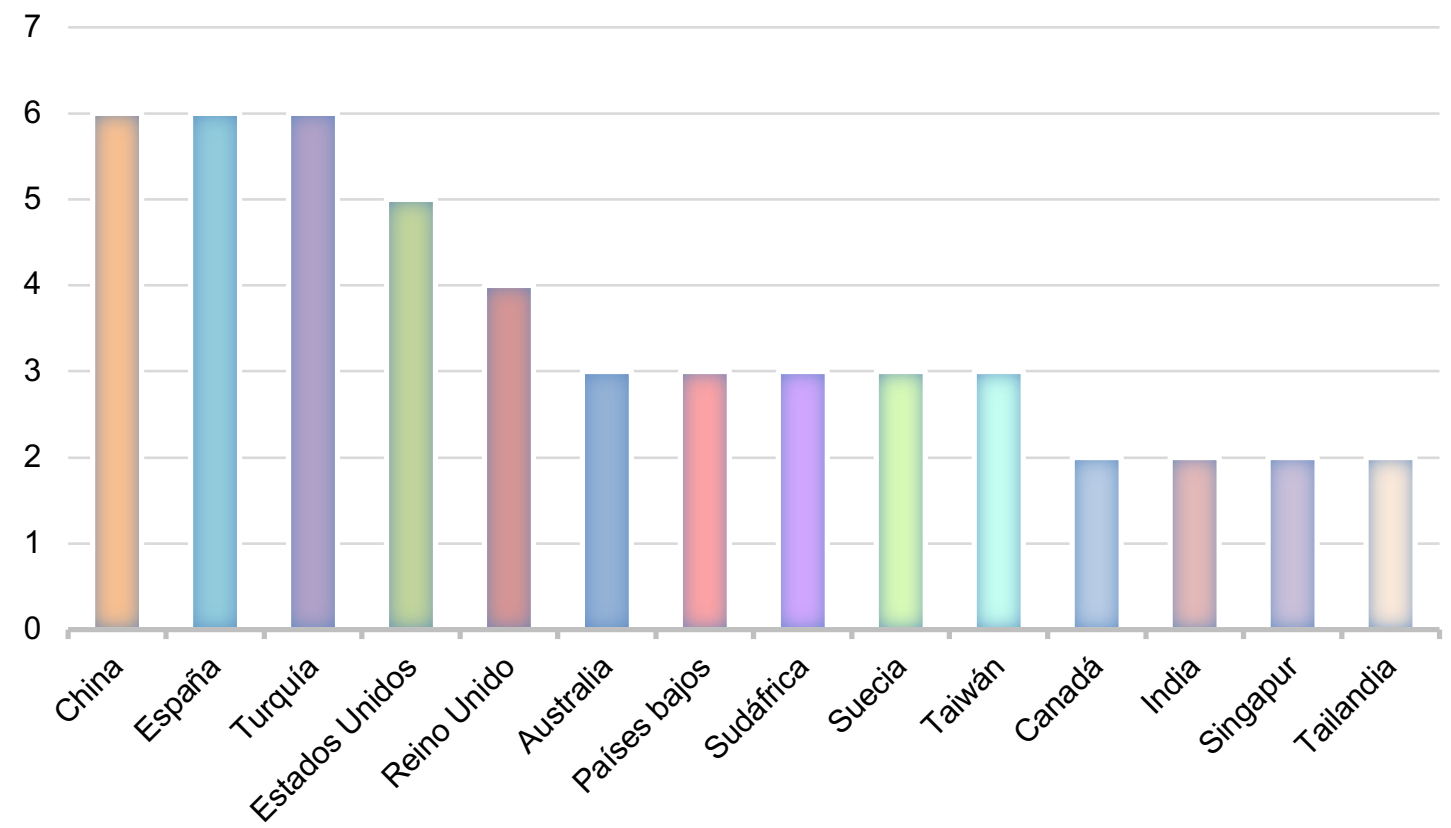

Figura 2. Número de publicaciones por país. 
Por lo que respecta a la colaboración y coautoría, en la Tabla 1 se han analizado el número de coautores para conocer la información concreta acerca de las estructuras y redes de colaboración en la comunidad científica, y con ello el carácter multidisciplinar (en mayor o menor medida) de las publicaciones estudiadas.

\begin{tabular}{|ccc|}
\hline & Tabla 1. Colaboración y coautoría. & \\
\hline Número de autores & $\mathbf{N}$ & $\%$ \\
\hline Uno & 16 & $27,11 \%$ \\
Dos & 17 & $28,81 \%$ \\
Tres & 13 & $22,03 \%$ \\
Más de tres & 13 & $22,03 \%$ \\
\hline
\end{tabular}

Los resultados muestran que los datos son bastante equilibrados, sin embargo, la mayoría de los documentos fueron escritos en colaboración (84\%). Por su parte, el $44 \%$ cuenta con autorías de tres o más. Si analizamos estos datos de forma longitudinal los resultados no son muy relevantes ya que se registran artículos de un solo autor tanto en los inicios como en la actualidad, del mismo modo que se contemplan trabajos con muchos coautores en los distintos años. Es de destacar que el documento con mayor número de firmas se ha escrito en 2014 (Miller et al., 2014) con diez autores, seguido de dos, uno en 2018 y otro 2007, con seis autores cada uno (Reddy, et al., 2018; Davis, et al. (falta indicar el año de publicación), lo que tampoco indica ningún incremento significativo de la colaboración entre autores a lo largo del tiempo.

Por último, por lo que se refiere a la repercusión e impacto de los artículos analizados, en la Tabla 2 se puede observar que, del grueso de las publicaciones revisadas, un $30,5 \%$ no tienen ninguna cita desde su publicación. Por su parte, el $32,2 \%$ conforma el total de los artículos que suman entre una y cinco citas, lo que supone una gran cantidad de trabajos con muy poca repercusión entre la comunidad científica. Sin embargo, es destacable también que un $13,55 \%$ cuente con más de veinte citas, como los trabajos de Rohaan, Taconis y Jochems (2010) y Davis, et al. (2008), habiendo sido citados 30 y 63 veces, respectivamente. El primero de estos trabajos es una revisión de la literatura en la que se concluye la relación existente entre el conocimiento tecnológico de los docentes y las actitudes positivas del alumnado hacia las tecnologías. El segundo de ellos es un estudio comparativo con grupo de intervención con aprendizaje con computadora y grupo control con enseñanza basada en exposición del docente, cuyos resultados indican la eficacia de ambas metodologías.

\begin{tabular}{|ccc|}
\hline \multicolumn{3}{c|}{ Tabla 2. Número de citas recibidas. } \\
\hline & $\mathbf{N}$ & $\%$ \\
\hline Ninguna & 18 & $30,5 \%$ \\
Una & 8 & $13,56 \%$ \\
Dos & 6 & $10,16 \%$ \\
Entre tres y cinco & 5 & $8,47 \%$ \\
De seis a diez & 9 & $15,25 \%$ \\
Más de 10 & 4 & $6,77 \%$ \\
20 o más & 6 & $10,17 \%$ \\
30 o más & 1 & $1,69 \%$ \\
Más de 60 & 1 & $1,69 \%$ \\
\hline
\end{tabular}

En segundo lugar, se han considerado variables estructurales y de contenido.

En cuanto a las variables estructurales, como en el estudio de Morales, Ortega, Conesa y Ruíz-Esteban (2017), se consideró las instituciones de procedencia de los coautores con el fin de determinar qué cooperaciones nacionales o internacionales se establecen a la hora de publicar sobre el tema. 


\begin{tabular}{|ccc|}
\hline \multicolumn{3}{|c|}{ Tabla 3. Procedencia de los coautores. } \\
\hline & $\mathbf{N}$ & $\%$ \\
\hline Sin coautores & 16 & $27,11 \%$ \\
Misma institución que el $1{ }^{\circ}$ autor & 17 & $28,81 \%$ \\
Institución del mismo país & 18 & $30,50 \%$ \\
Institución del extranjero (mismo continente) & 4 & $6,77 \%$ \\
Institución del extranjero (diferente continente) & 4 & $6,77 \%$ \\
\hline
\end{tabular}

A partir de los datos de la Tabla 3, se observa que, en la mayoría de los documentos, los coautores pertenecen a distintas instituciones del mismo país que el primer autor. En segundo lugar, destacan los artículos cuya autoría pertenece a la misma institución, entre el que encontramos el de Cózar, de Moya, Hernández y Hernández (2016) en el que la coordinación es interdepartamental, dentro de la misma universidad. Estos datos van sucedidos por ocho documentos cuyos autores han trabajado en colaboración desde distintos países, cuatro de ellos de diferente continente inclusive, lo que desvela una coordinación a gran escala, por ejemplo, entre Valencia y Estados Unidos (Roig, Mengual y Quinto, 2015), entre Reino Unido y Perú (Rivero, Chavez, Vasquez, y Blumen, 2016), entre Estados Unidos y Austria (Miller et al, 2014) o entre Reino Unido e Israel (Mor y Mogilevsky, 2013). Es de destacar que los artículos con mayores redes de cooperación internacional han sido publicados recientemente, en los últimos cinco años. También enfatizamos que parte de la autoría de uno de ellos pertenece a una universidad española, la Universitat de València en concreto y que, además, ha sido publicado en Comunicar, la segunda revista científica española con mayor impacto según ResearchGate tanto en Ciencias Sociales como en Comunicación (Poveda y Delgado, 2018).

Para el análisis de contenido como variable, se establecieron cinco grandes categorías con la pretensión de clasificar conceptualmente la producción científica obtenida, su distribución queda reflejada en la Figura 3. Las categorías determinadas fueron: la organización del sistema, las tipologías educativas, los agentes implicados, las tareas docentes y el uso de objetos didácticos.

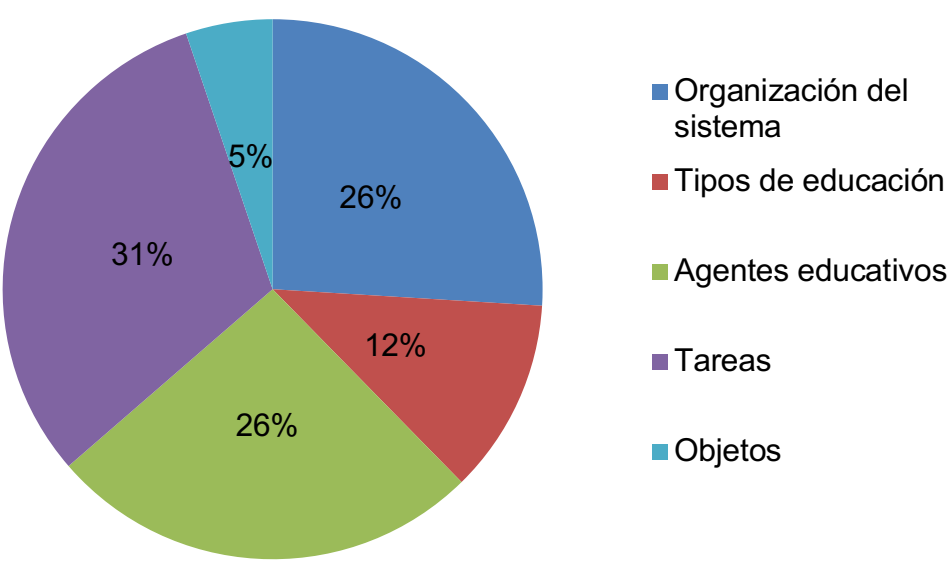

Figura 3. Distribución del contenido de los documentos

A partir de éstas se clasificaron los artículos en función de su temática principal desglosando los resultados en subcategorías (Tabla 4). Respecto a la primera, veinte de las publicaciones se refieren a las distintas etapas educativas, seis de ellos a educación primaria (Chiphiko y Shawa, 2014; Cyvin, 2013; Letchumanan, Muthusamy, Govindasamy y Farashaiyan, 2016; Mikerova, Sergeeva, Mardirosova, Kazantseva y 
Karpenko, 2018; Pareto, 2014; Sürmeli, Aşçi y Kizilhan, 2009), el segundo de los anteriores abarca también la educación secundaria, otros nueve se dedican a estudios universitarios en distintas disciplinas, tres de los cuales se centran en la formación de maestros (Cózar, de Moya, Hernández y Hernández, 2016; Gill, Dalgarno y Carlson, 2014 Llamas y Macías, 2018). Se registran cuatro documentos dedicados a la formación permanente a lo largo de todo el ciclo vital entre el que destacamos el de Zhang y Looi (2011) que se centra en el desarrollo de herramientas innovadoras sostenibles para el aprendizaje continuo. Ninguno de los artículos revisados tiene como temática predominante la educación infantil ni el bachillerato.

Por lo referido a la segunda categoría, hemos registrado siete estudios centrados en la educación formal (cinco de los cuales se agrupan entorno a áreas curriculares como las matemáticas, en Pitchford (2015), por ejemplo), uno en la no formal y uno en la informal. Ninguno de los documentos tiene como temática principal lo procedimental ni lo conceptual.

Respecto a los agentes implicados, de los veinte artículos dedicados a su estudio, catorce se centran en los docentes, los cuales han sido clasificados según el área específica de la docencia en la que se centraban: percepción de los docentes (McKenney y Voogt, 2017; Spiteri y Chang, 2018), prácticas docentes (Orlando, 2014), estrategias docentes (Liu, 2013), diseño de materiales (Altimparmak y Yazici, 2010), construcción del currículo (Kasapoğlu, 2010), formación inicial (un conjunto de siete artículos tratan este tema y destacamos el de Chambers (2002) por dedicarse a la formación específica necesaria para ser miembro del comité de tecnología en la escuela) y conocimientos docentes. Por su parte, cinco artículos más ofrecen investigaciones sobre el equipo directivo, entre los que se destacan el de Papaioannou y Charalambous (2011) por centrarse en el impacto de las actitudes y percepciones del director para la integración de las TIC y el de Aksal (2015) por la consideración del director como un posible líder digital para la cultura escolar. Por último, registramos un único documento sobre la familia, el de Smith, Tandon, Bair-Merritt y Hanson (2015) que solamente estudia las necesidades de los padres varones.

Pasando a la cuarta categoría, quince de los estudios se centran en innovación, entre los que podemos destacar el de Tang (2017) o Zhang y Looi (2011) sobre innovación organizativa, o el de Hopkins, Regehr y Wilson (2011) sobre entornos virtuales en un laboratorio de anatomía, o los de Hung, Kuo, Sun y Yu, (2014), Pareto (2014) y Reynolds, Baik y Li (2013) sobre gamificación. Cinco artículos se dedican a tareas de evaluación de resultados, algunos evalúan la motivación del alumnado (Wu y Tai, 2016), otros el rendimiento (Jang, 2015; Hung, Kuo, Sun y Yu, 2014), el interés (Sládek, Miér y Benárová, 2011) o los resultados de aprendizaje obtenidos por el alumnado (Günes y Dilek, 2009). Finalmente, un artículo estudia la alfabetización tecnológica (Nia y Vries, 2016), dos la atención a la diversidad (Brulé y Bailly, 2018 dedicado a alumnado con discapacidad visual; Cascales, Martínez, Pérez y Contero, 2017 acerca de las necesidades educativas especiales en general) y uno tareas de desarrollo (Ojo, Abayoi y Odozi, 2014).

Respecto a la última categoría sobre los objetos de aprendizaje se registran cuatro documentos en total, uno de ellos estudia aplicaciones (Tärning, 2018) y los otros tres soportes tales como los dispositivos móviles, mediante el modelo TPAK (Wong, Chai, Zhang y King, 2015), las tabletas (Pitchford, 2015) o el libro de texto (Sothayapetch, Lavonen y Juuti, 2013). 


\begin{tabular}{|lcl|}
\hline \multicolumn{3}{|c|}{ Tabla 4. Temática central del documento desglosada } \\
\hline \multirow{4}{*}{ Organización del sistema } & Infantil & 0 \\
& Primaria & 6 \\
& Secundaria & 1 \\
& Bachiller & 0 \\
& Universidad & 9 \\
& A lo largo de la vida & 4 \\
\hline \multirow{3}{*}{ Tipos de educación } & Formal & 7 \\
& No formal & 1 \\
& Informal & 1 \\
\hline \multirow{3}{*}{ Agentes } & Docentes & 14 \\
& Equipo directivo & 5 \\
& Familia & 1 \\
\hline \multirow{4}{*}{ Tareas } & Desarrollo & 1 \\
& Innovación & 15 \\
& Evaluación de resultados & 5 \\
Objetos & Alfabetización tecnológica & 1 \\
& Atención a la diversidad & 2 \\
\hline
\end{tabular}

\section{Conclusiones}

El objetivo del presente estudio fue analizar las tendencias en investigación sobre las TAC (Tecnologías del Aprendizaje y el Conocimiento) en el ámbito de las Ciencias Sociales. Para ello, se realizó un análisis bibliométrico de la producción científica depositada en la base de datos Scopus. Se han analizado una selección de los indicadores de productividad científica, colaboración, consumo y de repercusión e impacto, así como, variables estructurales y de contenido de los artículos disponibles.

Respecto al análisis de los indicadores, en primer lugar, los datos indican que el interés y esfuerzo por el estudio del impacto de las TAC ha crecido en los últimos tiempos, así se refleja en el aumento de trabajos publicados y disponibles en la base de datos Scopus. Además, entre los países con mayor producción científica en este campo destaca la posición predominante de España. Al centrar la mirada en las revistas en las que se publican estos artículos, tan sólo cinco artículos están escritos en lengua castellana. Los artículos publicados en español se encuentran en las revistas: Educare (Baltodano, 2018), Complutense de Educación (Llamas y Macías, 2018), Psicología (Perú) (Rivero, Chavez, Vasquez, y Blumen, 2016), Comunicar (Prendes, Castañeda, Gutiérrez, 2010) y Formación Universitaria (Cózar, de Moya, Hernández y Hernández, 2016). Al analizar las características de la coautoría a través del número de firmantes o el número medio de autores es preciso señalar que el grado de colaboración es alto.

Respecto a la temática, o grandes líneas de investigación los datos del presente estudio permiten identificar la existencia de cinco grandes líneas, que son la organización del sistema, las tipologías educativas, los agentes implicados, las tareas educativas y el uso de objetos didácticos digitales. Del análisis de los documentos seleccionados, se concluye que el proceso de integración de las tecnologías en las prácticas de enseñanza-aprendizaje en las diferentes etapas educativas es una realidad, principalmente en la escuela Primaria (Chiphiko y Shawa, 2014; Mikerova, Sergeeva, Mardirosova, Kazantseva, y Karpenko, 2018) y en educación Superior, tanto en la formación inicial de futuros profesionales de la educación (Gill, Dalgarno y Carlson, 2014) como de otros campos como la medicina (Qian y Huang, 2017) o una ingeniería (Chen, Liu, Qi y Geng, 2016). Todo esto sin olvidar la necesidad de formación a lo largo de toda la vida (Poitras, Doleck, Huang, Li y Lajoie, 2017). Pero va más allá de la 
educación formal, que incluye tanto el contenido curricular (Cascales, Martínez, Pérez y Contero, 2017) como la educación en valores, en cuestiones tan importantes como el cambio climático o los derechos de los niños (Makrakis, Gkotzos y Larios, 2012) y, además existen publicaciones centradas en la educación no formal (Intarat y Chanchalor, 2017) y en la informal (Erjavec, 2013). Como era de esperar, la mayor parte de las publicaciones se centran en los docentes (Liu, 2013) o en miembros del equipo directivo (Aksal, 2015) en cuanto que gestores o líderes. Tan sólo existe un estudio centrado en la familia (Smith, Tandon, Bair-Merritt y Hanson, 2015). Respecto a las tareas docentes incluyen fundamentalmente la innovación (Mkhize, Mtsweni y Buthelezi, 2016) y la atención a la diversidad (Brulé y Bailly, 2018), tan necesaria para el diseño de la respuesta educativa en un contexto inclusivo. Por último, parece evidente que las tecnologías han permitido aumentar la motivación e interés en el aprendizaje (Wu y Tai, 2016) y mejorar el rendimiento del alumnado (Jang, 2015).

En conclusión, muchas son las posibilidades de las TAC como herramientas para hacer posibles otras prácticas docentes, para activar otras estrategias, como la gamificación (Hung, Kuo, Sun y Yu, 2014) o el e-learning (Lumadi, 2014), ante la digitalización que se está produciendo desde finales del siglo XX.

Por lo que respecta a las líneas de investigación futura, queda reflexionar sobre el devenir de la educación en la sociedad digital para conseguir el máximo beneficio de la incorporación de la tecnología al proceso de enseñanza-aprendizaje. Tras la realización de este estudio, surgen nuevas preguntas de investigación como, por ejemplo, qué efectos tiene el uso de tecnología desde una perspectiva de género, cuál es el papel que deben asumir las familias en este proceso o cómo se diseñarán los planes de estudios del futuro para los profesionales de la educación, porque como se ha encontrado en estudios previos parece que, al menos en nuestro país, la tendencia es a abordar la formación inicial en competencia digital desde la transversalidad (Marín, Vidal, Peirats y San Martín, en prensa).

\section{Referencias}

Aksal, F. A. (2015). Are headmasters digital leaders in school culture? Egitim Ve Bilim, 40(182), 77-86. doi:10.15390/EB.2015.4534

Aleixandre, R. (2010). Bibliometría e indicadores de producción científica. En J. Jiménez, J.M. Argimon, A. Martín y T. Vilardell (Eds.), Publicación científica biomédica: cómo escribir $y$ publicar un artículo de investigación (p. 363-384). Barcelona: Editorial Elsevier.

Altiparmak, M., \& Yazici, N. N. (2010). Easy biotechnology: Practical material designs within team activities in learning biotechnological concepts \& processes. Paper presented at the Procedia - Social and Behavioral Sciences, 2(2) 4115-4119. doi:10.1016/j.sbspro.2010.03.649
Baltodano, M. (2018). Análisis e implicaciones de los resultados de las prácticas pedagógicas de un docente líder en una prueba piloto de innovación en el proyecto Conectándonos. Revista Electrónica Educare, 22(2) doi:10.15359/ree.22-2.4

Bell, D. (1973). The coming of post-industrial society: A venture in social forecasting. New York, USA: Basic Books.

Bordons, M., y Zulueta, M. (1999). Evaluación de la actividad científica a través de indicadores bibliométricos. Revista española de cardiología, 52(10), 790-800.

Brulé, E., \& Bailly, G. (2018). Taking into account sensory knowledge: The case of geotechnologies for children with visual impairments. Paper presented at the Conference on Human Factors in Computing Systems - Proceedings, 2018-April doi: $10.1145 / 3173574.3173810$ 
Cascales, A., Martínez, M., Pérez, D., \& Contero, M. (2017). Using an augmented reality enhanced tabletop system to promote learning of mathematics: A case study with students with special educational needs. Eurasia Journal of Mathematics, Science and Technology Education, 13(2), 355-380. doi:10.12973/eurasia.2017.00621

Castells, M. (1997). La era De La Información: Vol.1: La Sociedad Red. Madrid, Spain: Alianza.

Chambers, D. (2002). Developing school information technology leaders for the future. Journal of Information Technology for Teacher Education, 11(3), 303-314. doi:10.1080/14759390200200139

Chen, Z., Liu, L., Qi, X., \& Geng, J. (2016). Digital mining technology-based teaching mode for mining engineering. International Journal of Emerging Technologies in Learning, 11(10), 47-52. doi:10.3991/ijet.v11i10.6271

Chiphiko, E., \& Shawa, L. B. (2014). Implementing learner-centred approaches to instruction in primary schools in Malawi. Mediterranean Journal of Social Sciences, 5(23), 967-975. doi:10.5901/mjss.2014.v5n23p967

Cózar, R., de Moya, M. V., Hernández, J. A., y Hernández, J. R. (2016). Conocimiento y uso de las tecnologías de la información y las comunicaciones (TIC) según el estilo de aprendizaje de los futuros maestros. Formación Universitaria, 9(6), 105-118. doi:10.4067/S0718-50062016000600010

Cyvin, J. (2013). Challenges related to interdisciplinary use of digital mapping technology in primary and lower secondary schools. Norsk Geografisk Tidsskrift, 67(3), 162-171.

doi:10.1080/00291951.2013.804877

Davis, J., Chryssafidou, E., Zamora, J., Davies, D., Khan, K., \& Coomarasamy, A. (2007). Computer-based teaching is as good as face-to-face lecture-based teaching of evidence based medicine: $A$ randomised controlled trial. BMC Medical Education, 7 doi:10.1186/1472-6920-7-23

De Oliveira, G. P., \& Dias, M. (2018). Constructions in plane euclidean geometry: The perspectives opened by didactic strategies with technologies. Bolema Mathematics Education Bulletin, 32(60), 92116. doi: 10.1590/1980-4415v32n60a05

Drucker, P. F. (1969). The age of discontinuity. Guidelines to our changing society. New York, USA: Harper \& Row.
Enríquez, S. (2012) Luego de las TIC, LAS TAC. Ponencia presentada en las II Jornadas Nacionales de TIC e Innovación en el Aula. Recuperado http://sedici.unlp.edu.ar/bitstream/handle/10 915/26514/ponencia ead enriquez silvia cecilia.luego+de+las+TIC,+las+TAC+(1).pdf ?sequence $=1$

Erjavec, K. (2013). Informal learning through facebook among slovenian pupils. Comunicar, 21(41), 117-126. doi:10.3916/C41-2013-11

García-Peñalvo, F. J. (2018). La Universidad en la Sociedad del Conocimiento. Grupo GRIAL. Disponible en http://repositorio.grial.eu/handle/grial/1191

Gill, L., Dalgarno, B., \& Carlson, L. (2014). How does pre-service teacher preparedness to use ICTs for learning and teaching develop through their degree program? Australian Journal of Teacher Education, 40(1), 36-60. doi:10.14221/ajte.2015v40n1.3

Granda, J. I., Alonso, A., García, F., Solano, S., Jiménez, C. A., \& Aleixandre, R. (2013). Ciertas ventajas de Scopus sobre Web of Science en un análisis bibliométrico sobre tabaquismo. Revista española de documentación científica, 36(2), 1-11.

Güneş, T., \& Dilek, N. S. (2009). Evaluation of science and technology program according to students' opinions. Procedia - Social and Behavioral Sciences, 1(1), 1396-1401. doi:10.1016/j.sbspro.2009.01.246

Hernández, V., Sans, N., Jové, M. C., \& Reverter, J. (2016). Comparación entre Web of Science y Scopus, estudio bibliométrico de las revistas de anatomía y morfología. International Journal of Morphology, 34(4), 1369-1377.

Hopkins, R., Regehr, G., \& Wilson, T. D. (2011). Exploring the changing learning environment of the gross anatomy lab. Academic Medicine, 86(7), 883-888. doi:10.1097/ACM.0b013e31821de30f

Hung, C., Kuo, F., Sun, J. \& Yu, P. (2014). An interactive game approach for improving students' learning performance in multi-touch game-based learning. Transactions on Learning Technologies, 7(1), 31-37. doi:10.1109/TLT.2013.2294806

Intarat, S., \& Chanchalor, S. (2017). ICTs for nonformal education in rural Thailand. Australasian Journal of Educational Technology, 33(4), 107-121. doi:10.14742/ajet.3165 
Jaén, M. D. M., García, A. P., \& Piñar, J. L. A. (2012). Las TIC en la formación inicial y en la formación permanente del profesorado de infantil y primaria. Edutec. Revista Electrónica de Tecnología Educativa, (41), 197-211.

Jang, Y. (2015). Convenience matters: A qualitative study on the impact of use of social media and collaboration technologies on learning experience and performance in higher education. Education for Information, 31(2), 73-98. doi: 10.3233/EFI-150948

Kasapoğlu, K. (2010). A qualitative analysis: How is elementary reading and writing curriculum constructed by first graders? Paper presented at the Procedia - Social and Behavioral Sciences, 2(2) 996-1001. doi: 10.1016/j.sbspro.2010.03.140

King, J. (1987). A review of bibliometric and other science indicators and their role in research evaluation. Journal of information science, 13(5), 261-276.

Letchumanan, K., Muthusamy, P., Govindasamy, P., \& Farashaiyan, A. (2016). Online interactive activities to learn ramayana epic by primary tamil students. Asian Social Science, 12(5), 201-207. doi:10.5539/ass.v12n5p201

Leung, C. (2010). Critical factors of implementing knowledge management in school environment: A qualitative study in Hong Kong. Research Journal of Information Technology, 2(2), 66-80. doi:10.3923/rjit.2010.66.80

Liu, S. (2013). Exploring the instructional strategies of elementary school teachers when developing technological, pedagogical, and content knowledge via a collaborative professional development program. International Education Studies, 6(11), 5868. doi:10.5539/ies.v6n11p58

Llamas, F., \& Macías, E. (2018). Formación inicial de docentes en educación básica para la generación de conocimiento con las tecnologías de la información y la comunicación. Revista Complutense de Educación, 29(2), 577-593. doi:10.5209/RCED. 53520

Lozano, R. (2011). De las TIC a las TAC: tecnologías del aprendizaje y del conocimiento. Anuario ThinkEPI, 1(1), 45-47.

Lumadi, M. W. (2014). Challenges besetting teachers in the implementation of e-learning in schools: A guide to curriculum development. Mediterranean Journal of Social Sciences, 5(20), 2814-2822. doi:10.5901/mjss.2014.v5n20p2814
Machlup, F. (1962). The production and distribution of knowledge in the United States. Princeton, N.J., USA: Princeton University Press.

Makrakis, V., Gkotzos, D., \& Larios, N. (2012). ICT-enabled climate change education and children's rights. Journal of Teacher Education for Sustainability, 14(2), 89-110. doi:10.2478/v10099-012-0011-y

Marín, D., Vidal, M.I., Peirats, J. y San Martín, A. (en prensa). Competencia Digital transversal en la formación del profesorado, análisis de una experiencia. Revista Innoeduca.

McKenney, S., \& Voogt, J. (2017). Expert views on TPACK for early literacy: Priorities for teacher education. Australasian Journal of Educational Technology, 33(5), 1-14. doi:10.14742/ajet.2502

Mikerova, G., Sergeeva, B., Mardirosova, G., Kazantseva, V., \& Karpenko, A. (2018). Learning environment affecting primary school student's mental development and interest. International Electronic Journal of Elementary Education, 10(4), 407-412. doi:10.26822/iejee.2018438130

Miller, B. T., Singh, R. P., Schalk, V., Pevzner, Y., Sun, J., Miller, C. S., Boresch, S. Ichiye, T. Brooks, B. R. \& Woodcock III, H. L. (2014). Web-based computational chemistry education with CHARMMing I: Lessons and tutorial. PLoS Computational Biology, 10(7) doi: 10.1371/journal.pcbi.1003719

Ministerio de Educación (2013). Ley orgánica para la mejora de la calidad educativa (LOMCE) (Ley Orgánica 8/2013, 9 de diciembre). Boletín Oficial del Estado, $n^{\circ} 295$, 10 diciembre.

Mkhize, P., Mtsweni, S., \& Buthelezi, P. (2016). Diffusion of innovations approach to the evaluation of learning management system usage in an open distance learning institution. International Review of Research in Open and Distance Learning, 17(3), 295312. doi:10.19173/irrodl.v17i3.2191

Mor, Y., \& Mogilevsky, O. (2013). The learning design studio: Collaborative design inquiry as teachers' professional development. Research in Learning Technology, 21 doi:10.3402/rlt.v21i0.22054

Mori, S., \& Shiogama, H. (2018). The value of knowledge accumulation on climate sensitivity uncertainty: Comparison between perfect information, single stage and act then learn decisions. Sustainability Science, 13(2), 351-368. doi:10.1007/s11625-0180528-7 
Muñoz, J. M. (2008). NNTT, TIC, NTIC, TAC... en educación ¿pero esto qué es? Quaderns digitals: Revista de Nuevas Tecnologías y Sociedad, 51, 43-60.

Nia, M. G., \& de Vries, M. J. (2016). 'Standards' on the bench: Do standards for technological literacy render an adequate image of technology? Journal of Technology and Science Education, 6(1), 5-18. doi:10.3926/jotse.207

Ojo, L. B., Abayomi, A. A., \& Odozi, A. F. (2014). Quality education: A harbinger for attaining millennium development goals in Nigeria. Mediterranean Journal of Social Sciences, 5(27), 861-869. doi:10.5901/mjss.2014.v5n27p861

Orlando, J. (2014). Teachers' changing practices with information and communication technologies: An up-close, longitudinal analysis. Research in Learning Technology, 22, 14-37. doi:10.3402/rlt.v22.21354

Papaioannou, P., \& Charalambous, K. (2011). Principals' attitudes towards ICT and their perceptions about the factors that facilitate or inhibit ICT integration in primary schools of cyprus. Journal of Information Technology Education: Research, 10(1), 349-369. doi:10.28945/1530

Pareto, L. (2014). A teachable agent game engaging primary school children to learn arithmetic concepts and reasoning. International Journal of Artificial Intelligence in Education, 24(3), 251-283. doi:10.1007/s40593-014-0018-8

Perianes-Rodriguez, A., Waltman, L., \& van Eck, N. J. (2016). Constructing bibliometric networks: A comparison between full and fractional counting. Journal of Informetrics, 10(4), 1178-1195.

Phungsuk, R., Viriyavejakul, C., \& Ratanaolarn, T. (2017). Development of a problem-based learning model via a virtual learning environment. Kasetsart Journal of Social Sciences, 38(3), 297-306. doi:10.1016/j.kjss.2017.01.001

Pitchford, N. J. (2015). Development of early mathematical skills with a tablet intervention: A randomized control trial in Malawi.

Roig, R., Mengual, S., \& Quinto, P. (2015). Primary teachers' technological, pedagogical and content knowledge. Comunicar, 23(45), 151-159. doi:10.3916/C45-2015-16.
Frontiers in Psychology, doi:10.3389/fpsyg.2015.00485

Poitras, E., Doleck, T., Huang, L., Li, S., \& Lajoie, S. (2017). Advancing teacher technology education using open-ended learning environments as research and training platforms. Australasian Journal of Educational Technology, 33(3), 32-45. doi:10.14742/ajet.3498

Poveda, I. y Delgado, E. (2018). Impacto de las revistas científicas españolas según ResearchGate (2000-2015). Granada, 16 de noviembre de 2018.

Prendes, M. P., Castañeda, L., \& Gutiérrez, I. (2010). Competencias para el uso de TIC de los futuros maestros. Comunicar, 17(35), 175-181. doi:10.3916/C35-2010-03-11

Qian, Z. \& Huang, G. (2017). Human capital and innovation ability in medical education: An empirical study. Eurasia Journal of Mathematics, Science and Technology Education, 13(8), 5395-5403. doi:10.12973/eurasia.2017.01008a

Reddy, J., Jagadish, Charanraj, N., Praveen, M. N., Sai, Y. D., \& Sathish, B. (2018). Factors affecting implementation of green supply chain management in tech manora packing: A case study. International Journal of Engineering and Technology (UAE), 7(3), 171-174. doi:10.14419/ijet.v7i3.12.15912

Reynolds, R. B., Baik, E., \& Li, X. (2013). Collaborative information seeking in the wild: Middle-schoolers' self-initiated teamwork strategies to support game design. Proceedings of the ASIST Annual Meeting, 50(1) doi:10.1002/meet.14505001083

Rivero, C., Chavez, A., Vasquez, A., \& Blumen, S. (2016). Las TIC en la formación universitaria. Logros y desafíos para la formación en psicología y educación. Revista de Psicología (Perú), 34(1), 185-199. doi:10.18800/psico.201601.007

Rohaan, E. J., Taconis, R., \& Jochems, W. M. G. (2010). Reviewing the relations between teachers' knowledge and pupils' attitude in the field of primary technology education. International Journal of Technology and Design Education, 20(1), 15-26. doi:10.1007/s10798-008-9055-7

Salvat G. y Serrano, V. (2011). La revolución digital y la sociedad de la información. Manganeses de la Lampreana, Zamora: Comunicación Social.

Sancho, J. M. (2008). De TIC a TAC, el difícil tránsito de una vocal. Revista de Investigación en la Escuela, 64, 19-30. 
Shahali, E. H. M., \& Halim, L. (2010). Development and validation of a test of integrated science process skills. Paper presented at the Procedia - Social and Behavioral Sciences, 9, 142-146. doi: 10.1016/j.sbspro.2010.12.127

Sládek, P., Milér, T., \& Benárová, R. (2011). How to increase students' interest in science and technology. Procedia - Social and Behavioral Sciences, 12 , 168-174. doi:10.1016/j.sbspro.2011.02.024

Smith, T. K., Tandon, S. D., Bair-Merritt, M. H., \& Hanson, J. L. (2015). Parenting needs of urban, african american fathers. American Journal of Men's Health, 9(4), 317-331. doi:10.1177/1557988314545380

Sothayapetch, P., Lavonen, J., \& Juuti, K. (2013). An analysis of science textbooks for grade 6 : The electric circuit lesson. Eurasia Journal of Mathematics, Science and Technology Education, 9(1), 59-72. doi:10.12973/eurasia.2013.916a

Spiteri, M., \& Chang, S. (2018). Literature review on the factors affecting primary teachers' use of digital technology. Technology, Knowledge and Learning, 1, 343-358. doi: 10.1007/s10758-018-9376-x

Sürmeli, H., Aşçi, M., \& Kizilhan, P. (2009). Determination of the relationship between the samples of instructional methods and techniques prepared for 4th and 5th grade primary school science and technology curriculum and the principles of situated learning in turkey. Procedia - Social and Behavioral Sciences, 1(1), 483-488. doi:10.1016/j.sbspro.2009.01.088

Tang, H. (2017). Effects of leadership behavior on knowledge management and organization innovation in medicine and health sciences. Eurasia Journal of Mathematics, Science and Technology Education, 13(8), 5425-5433. doi:10.12973/eurasia.2017.00840a

Tärning, B. (2018). Review of feedback in digital applications - does the feedback they provide support learning? Journal of Information Technology Education: Research, 17, 247283. doi:10.28945/4104
Tomás-Górriz, V., \& Tomás-Casterá, V. (2018). La Bibliometría en la evaluación de la actividad científica. Hospital a Domicilio, 2(4), 145-163.

Touraine, A. (1969). La société post-industrielle. Paris: Denoël.

UNESCO. (2005). Towards Knowledge Societies. Paris, France: UNESCO Publishing. Disponible en: https://goo.gl/yzc6KX.

Urueña, S. (2016). Dimensiones de la inclusión de las TIC en el currículo educativo: una aproximación teórica. Teoría de la educación, 28(1), 209-223. doi: $10.14201 /$ teoredu2016281209223

Van Eck, N. J., \& Waltman, L. (2011). Text mining and visualization using VOSviewer. arXiv preprint arXiv:1109.2058.

Vyas, A. (2014). Geomatics education: Need assessment. Paper presented at the International Archives of the Photogrammetry, Remote Sensing and Spatial Information Sciences - ISPRS Archives, $\quad X L, \quad 8(1), \quad 1265-1267 . \quad$ doi: 10.5194/isprsarchives-XL-8-1265-2014 Retrieved from www.scopus.com

Wong, L., Chai, C., Zhang, X. \& King, R. (2015). Employing the TPACK framework for researcher-teacher co-design of a mobileassisted seamless language learning environment. Transactions on Learning $\begin{array}{lll}\text { Technologies, } & 8(1), & 31-42 .\end{array}$ doi:10.1109/TLT.2014.2354038

Wu, T. \& Tai, Y. (2016). Effects of multimedia information technology integrated multisensory instruction on students' learning motivation and outcome. Eurasia Journal of Mathematics, Science and Technology Education, 12(4), 1065-1074. doi:10.12973/eurasia.2016.1552a

Zhang, B. H., \& Looi, C. (2011). Developing a sustainable education innovation for seamless learning. Procedia - Social and Behavioral Sciences, 15, 2148-2154. doi:10.1016/j.sbspro.2011.04.069 